\title{
Community-acquired pneumonia and pneumococcal vaccination in the elderly
}

\author{
Charles Yin (MD/PhD 2021), Jeffrey Law (Meds 2016) \\ Reviewer: Dawn Bowdish, PhD (Department of Molecular Medicine, McMaster University)
}

\begin{abstract}
Community-acquired pneumonia (CAP), most often caused by infection with the Gram-positive diplococcus Streptococcus pneumoniae, remains a leading cause of death in Canada amongst the elderly. With an aging population in Canada, CAP will soon be a significant challenge to the healthcare system in this country. In this article, we review the characteristics of CAP in the elderly, including its epidemiology, etiology and clinical features. We then provide an overview and history of pneumococcal vaccines and present current recommendations for $S$ pneumoniae vaccination in Canada.
\end{abstract}

\section{BACKGROUND}

Pneumonia, primarily a result of colonization and infection by the Gram-positive bacterium Streptococcus pneumoniae, remains a dangerous disease with a high mortality rate, especially amongst the elderly., Each year, approximately 200000 people in Canada contract pneumonia ${ }^{2}$ and around 5000 of those will die from pneumonia or complications related to pneumonia. ${ }^{3}$

Pneumonia can generally be divided into two major categories: community-acquired pneumonia (CAP) and hospital-acquired pneumonia (HAP). ${ }^{4}$ Nursing homes, where a significant portion of the elderly population in Canada resides, are considered community settings in the context of community- versus hospital-acquired pneumonia. ${ }^{4}$ We will focus on CAP as a majority of elderly patients with pneumonia contract their illness while in a community or in nursing homes. ${ }^{1,4}$

\section{EPIDEMIOLOGY OF COMMUNITY-ACQUIRED PNEUMONIA}

The overall incidence of CAP in Canada is estimated to be between 5.60 and 6.11 per 1000 people. $^{2}$ A 2-year study at an emergency department in Edmonton, Alberta found that incidence was significantly correlated with age. ${ }^{2}$ Incidence was found to increase with patient age for patients over 50, reaching a maximum of 46.39 per 1000 in patients 90 and older. Incidence was also found to be higher in men than in women in patients over the age of $70 .^{2} \mathrm{CAP}$ is also the 7 th leading cause of death in Canada, ${ }^{5}$ with an estimated mortality rate of between 0.95 and 11.7 per $1000 .{ }^{3}$

\section{ETIOLOGY OF COMMUNITY-ACQUIRED PNEUMONIA}

Microbes present in the upper airways routinely enter the lower airways. In the majority of cases, they are cleared by specific and nonspecific host defense mechanisms, but when this clearance fails to occur, pneumonia can result., ${ }^{1,4}$ Diverse factors that predispose individuals to developing pneumonia, and these can be broadly di- vided into host immunocompromise, exposure to a particularly virulent respiratory microbe or inoculation with an overwhelmingly large microbial load that overwhelms the immune system. ${ }^{4}$

The diagnosis of CAP in the primary care setting is for the most part a clinical diagnosis, and the infectious etiology is often poorly established. ${ }^{6}$ In a review of the literature, it was found that a definitive microbial agent was established in only $24-67 \%$ of CAP cases. ${ }^{6-10}$ Nevertheless, most studies agree that $S$ pneumoniae is the most common infectious agent responsible for CAP, being identified in 27.4 to $41.9 \%$ of CAP cases where a microbial etiology could be established..$^{6-10}$

\section{PNEUMOCOCCAL PNEUMONIA IN THE ELDERLY Risk factors}

The elderly are at increased risk of developing pneumococcal pneumonia. ${ }^{1,2}$ Specific risk factors that predispose the elderly to becoming colonized by $S$ pneumoniae include a depressed immune system, increased prevalence of comorbidities, especially lung and cardiovascular disease, and difficulty swallowing and aspiration. ${ }^{1}$

\section{Clinical presentation}

The clinical presentation of pneumonia in the elderly differs from the "typical" presentation of a young adult with pneumonia.,11 In particular, elderly patients with pneumonia tend to display fewer, or more subtle symptoms, with the authors of one study finding an average of 2.9-3.3 fewer reported symptoms in patients over 65 years of age. ${ }^{11}$ In particular, there is a lower incidence of fever in elderly patients with pneumonia. ${ }^{11}$ In contrast, there are some clinical comorbidities strongly associated with pneumonia in the elderly. Most significant amongst these is dementia, which is present in up to $41.1 \%$ of elderly patients with pneumonia. ${ }^{12}$

\section{Management}

Clinical guidelines recommend that elderly patients with pneumonia be managed differently. All elderly patients presenting with pneumonia should be assessed for clinical severity, using either the CURB-65 score (presence of confusion, BUN > 7mmol/L, respiratory rate $\geq 30$, systolic blood pressure $<90$ and/or diastolic blood pressure $<60$, age $\geq 65)^{13}$ or the Pneumonia Severity Index (PSI) developed by Fine et al, ${ }^{14}$ which is a more detailed guideline to assessing severity of pneumonia.

The Canadian Thoracic Society currently does not recommend different antibiotic regimens based on age. However, its most recent guidelines specifically recommend treating nursing home residents with either a respiratory fluoroquinolone alone or with amoxicillin-clavulin and a macrolide as the first-line empiric therapy. ${ }^{13}$ 


\section{STREPTOCOCCUS PNEUMONIAE VACCINOLOGY \\ Overview}

Pneumococcal vaccines come in 2 general categories. ${ }^{15,16}$ First are the older polysaccharide vaccines, which consist of purified capsule antigens. ${ }^{15,16}$ These polysaccharide capsule antigens are recognized by $\mathrm{B}$ cell receptors, resulting in the generation of anticapsule IgM antibodies. ${ }^{16}$ There are a number of distinct drawbacks to this strategy, including a lack of $\mathrm{T}$ cell antigen recognition and resultant lack of isotype switching and production of the IgA antibodies that would be needed to confer mucosal immunity against $S$ pneumoniae. $^{16}$

The newer generation of conjugated pneumococcal vaccines consist of capsule antigen conjugated to an immunogenic diphtheria toxoid. ${ }^{15,16}$ This leads to recognition by Th2 cells and the induction of isotype switching by B cells and the production of both IgA and IgG antibodies, and a stronger memory B cell response. ${ }^{16}$ In particularly, production of IgG is thought to be essential in the prevention of invasive pneumococcal disease. ${ }^{16}$ Unfortunately, the conjugated vaccine currently only contains a limited number of serotypes as compared to the newest generation of polysaccharide vaccine.

\section{History of pneumococcal vaccines}

Pneumococcal pneumonia and its complications were major sources of mortality before the widespread availability of antibiotics. Mortality rates in the 1920s and 1930s from pneumococcal pneumonia and associated bacteremia and meningitis were well in excess of $70 \%$ in hospitalized patients. ${ }^{15}$

Early efforts to develop a pneumococcal vaccine began in 1914 when British immunologist Sir Almroth Wright experimented with the use of a crude whole cell vaccine in South African miners, amongst whom pneumonia was epidemic. ${ }^{17}$ By the 1930 s scientists had advanced to the use of simple polysaccharide vaccines in small observational studies. ${ }^{15}$ These studies paved the way for a number of landmark controlled trials on bivalent, trivalent and tetravalent pneumococcal polysaccharide vaccines in the 1940s that showed for the first time that vaccination resulted in a drop in the incidence of pneumococcal pneumonia. . $^{18,19}$

However, by the 1950s the attitude of the public and regulatory bodies towards pneumococcal vaccines began to change with the widespread availability of antibiotics. ${ }^{15}$ Pneumococcal vaccination was thought to be unnecessary by regulators and it wasn't until the release of a landmark 1964 study by American infectious disease specialist Robert Austrian showing continued high mortality rates amongst patients with pneumonia that interest in a pneumococcal vaccine returned. ${ }^{15,20}$ Austrian himself was at the forefront of $S$ pneumoniae vaccinology, creating the PPSV14 pneumococcal vaccine, a polysaccharide vaccine against the 14 most common $S$ pneumoniae serotypes of the time, in $1970 .^{21}$ PPSV14 was adopted for widespread use in the United States by 1977 until 1983, when it was replaced by the PPSV23 vaccine, which expanded coverage to $23 \mathrm{~S}$ pneumoniae serotypes. ${ }^{15}$

\section{Currently available vaccines}

There are two pneumococcal vaccines currently available in the United States and Canada. The first of these is PPSV23 (Pneumovax), a polysaccharide vaccine covering 23 serotypes, produced by Merck. ${ }^{15,16}$ PPSV23 has been in used in the United States and Canada for several decades and a number of studies of its efficacy and safety have been published. A 2013 Cochrane review found that available evidence showed PPSV23 was effective at prevention of invasive pneumococcal disease (eg bacteremia, meningitis) but not pneumococcal pneumonia. ${ }^{22}$

The second vaccine available is PCV13 (Prevnar-13), a conjugate vaccine covering 13 serotypes, produced by Pzifer. ${ }^{15,16}$ Conjugated vaccines for $S$ pneumoniae have been available since 2000 in the form of PCV7, an early conjugate vaccine that provided coverage of $7 S$ pneumoniae serotypes. In 2011, PCV7 was replaced by the newer PCV13, ${ }^{15}$ which was made available in the United States under the US FDA's Early Access program, reflecting the changing paradigm of the pneumococcal vaccine from being thought of as unnecessary by regulators to now being recognized as essential in the reduction of pneumonia-related mortality. A large-scale clinical trial in nearly 85000 patients (the CAPiTA study) sponsored by Pzifer showed that PCV13 was 70\% effective at the prevention of invasive pneumococcal disease and $46 \%$ effective at the prevention of pneumococcal pneumonia. ${ }^{23}$

\section{CURRENT RECOMMENDATIONS ON S PNEUMONIAE VACCINATION IN THE ELDERLY}

Current recommendations on vaccination of the elderly against $S$ pneumoniae are based on those released by the US Advisory Committee on Immunization Practices (ACIP). ${ }^{24,25}$ For individuals 65 or older, the ACIP has recommended the administration of a single dose of PCV13 followed by a single dose of PPSV23 after 6-12 months. ${ }^{24}$ There is currently no evidence to suggest that revaccination with PPSV23 provides additional benefits. ${ }^{25}$

\section{CONCLUSION}

Pneumococcal pneumonia continues to be a major source of mortality for the elderly. The development of a vaccination program against $S$ pneumoniae has been a major step forward in the efforts to mitigate the impact of pneumonia on the health of the elderly.

\section{REFERENCES}

1. Loeb, M. Pneumonia in older persons. Clin Infect Dis. 2003 Nov;37(10):1335-9.

2. Marrie TJ, Huang JQ. Epidemiology of community-acquired pneumonia in Edmonton, Alberta: an emergency department-based study. Can Respir J. 2005 Apr;12(3):139-42.

3. Statistics Canada. Table 102-0552 Deaths and mortality rate, by selected grouped causes and sex, Canada, provinces and territories, annual. CANSIM (database). 2014 Jan 27.

4. Marrie TJ. Epidemiology, pathogenesis, and microbiology of community-acquired pneumonia in adults [Internet]. UpToDate; 2014 [cited 2014 Apr 19]. Available from: http://www.uptodate.com/contents/ epidemiology-pathogenesis-and-microbiology-of-community-acquired-pneumonia-in-adults. 
5. File TM Jr, Marrie TJ. Burden of community-acquired pneumonia in North American adults. Postgrad Med. 2010 Mar;122(2):130-41.

6. Johansson N, Kalin M, Tiveljung-Lindell, A, Giske CG, Hedlund J. Etiology of community-acquired pneumonia: increased microbiological yield with new diagnostic methods. Clin Infect Dis. 2010 Jan;50(2):202-9.

7. Restrepo MI, Mortensen EM, Velez JA, Frei C, Anzueto A. A comparative study of community-acquired pneumonia patients admitted to the ward and the ICU. Chest. 2008 Mar;133(3):610-7.

8. Cillóniz C, Ewig S, Polverino E, Marcos MA, Esquinas C, Gabarrus A, Mensa J, Torres A. Microbial aetiology of community-acquired pneumonia and its relation to severity. Thorax. 2011 Apr;66(4):340-6.

9. Shibli F, Chazan B, Nitzan O, Flatau E, Edelstein H, Blondheim O, Raz R, Colodner R. Etiology of community-acquired pneumonia in hospitalized patients in northern Israel. Isr Med Assoc J. 2010 Aug;12(8):477-82.

10. Song JH, Oh WS, Kang CI, et al. Epidemiology and clinical outcomes of community-acquired pneumonia in adult patients in Asian countries: a prospective study by the Asian network for surveillance of resistant pathogens. Int J Antimicrob Agents. 2008 Feb;31(2):107-14.

11. Metlay JP, Schulz R, Li YH, Singer DE, Marrie TJ, Coley CM, Hough LJ, Obrosky DS, Kapoor WN, Fine MJ. Influence of age on symptoms at presentation in patients with community-acquired pneumonia. Arch Intern Med. 1997 Jul;157(13):1453-9.

12. Mitchell SL, Teno JM, Kiely DK, Shaffer ML, Jones RN, Prigerson HG, Volicer L, Givens JL, Hamel MB. The clinical course of advanced dementia. N Engl J Med. 2009 Oct;361(16):1529-38.

13. Mandell LA, Marrie TJ, Grossman RF, Chow AW, Hyland RH, Canadian CAP Working Group. Summary of Canadian gyidelines for the initial management of community-acquired pneumonia: an evidence-based update by the Canadian Infectious Disease Society and the Canadian Thoracic Society. Can J Infect Dis. 2000 Oct;11(5):23748.

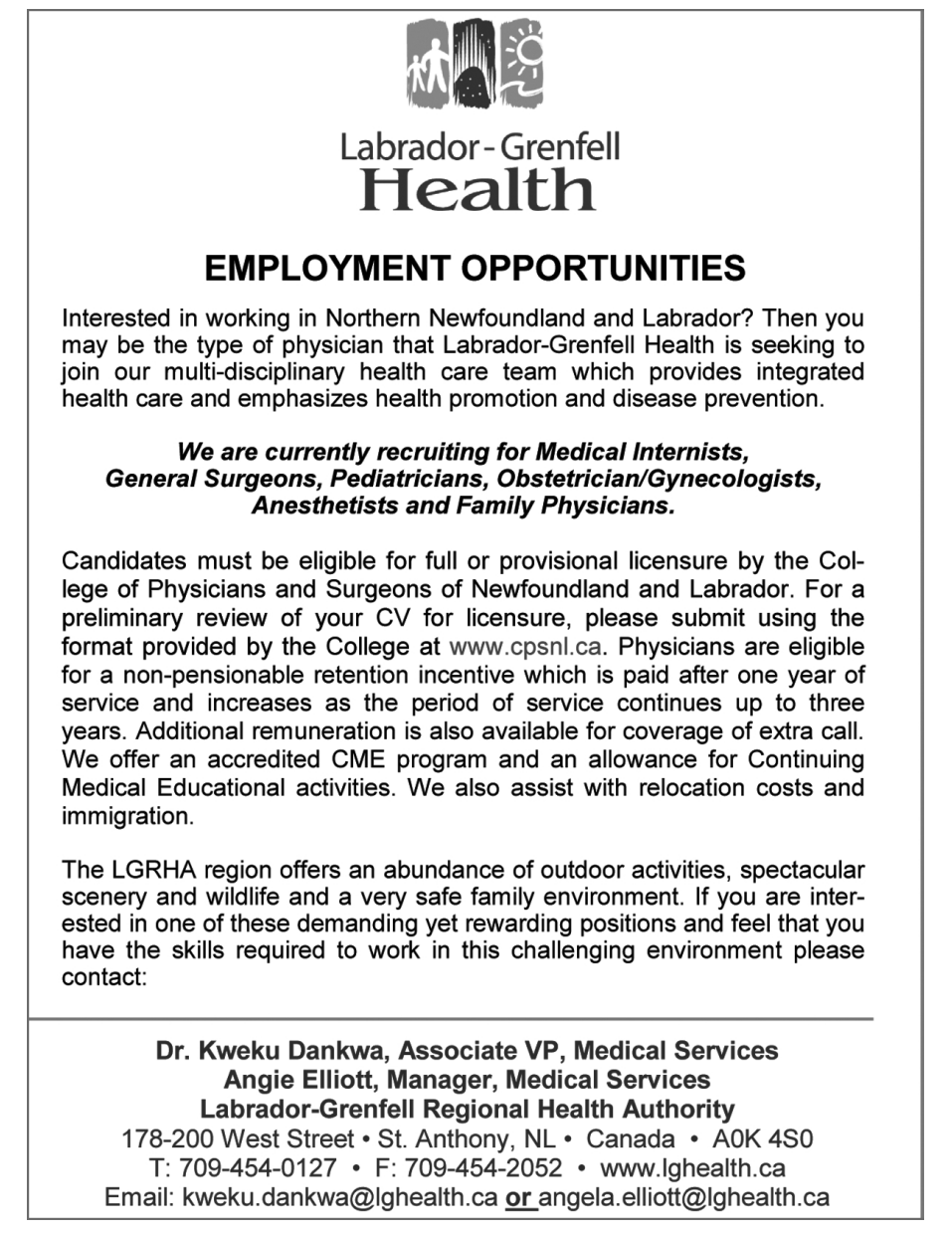

